\title{
DESIGN OPTIMISATION OF ELECTROMAGNETIC DEVICES USING CONTINUUM DESIGN SENSITIVITY ANALYSIS COMBINED WITH COMERCIAL EM SOFTWARE
}

\author{
Dong-Hun Kim ${ }^{1}$, Jan K. Sykulski ${ }^{2}$ and David A. Lowther ${ }^{3}$ \\ ${ }^{1}$ Kyungpook National University, South Korea \\ ${ }^{2}$ University of Southampton, United Kingdom \\ ${ }^{3}$ McGill University, Canada
}

\begin{abstract}
This paper deals with two kinds of optimisation problems, relevant to the optimised source distribution and the shape optimum design, using Continuum Design Sensitivity Analysis (CDSA) in combination with standard electromagnetic (EM) software. Fast convergence and compatibility with existing EM software are the distinctive features of the proposed implementation. In order to verify the advantages and also to facilitate understanding of the method itself, two design optimisation problems have been tested: one is an MRI design problem related to finding an optimal permanent magnet distribution and the other is a pole shape design problem of a BLDC motor for reducing cogging torque, using both $2 \mathrm{D}$ and $3 \mathrm{D}$ models.
\end{abstract}

\section{INTRODUCTION}

The concept of CDSA was introduced in the late 1980 's and successfully applied to the design of EM devices. However, so far most of the studies have been carried out separately from general-purpose EM software packages even though it is known that CDSA could provide an easy way of interfacing with them. Recently, the physical meaning of pseudo sources of an adjoint system in a CDSA when applied to shape optimisation was explored [1] and the approach was reported to avoid the need to access the source code of commercial programs. Moreover, the computing times required to find an optimal solution are not affected by the number of design variables. The initial, very encouraging, results have prompted researchers to pursue this technique further as it appears to be competitive compared, for example, with stochastic methods.

In this paper, two different optimisation problems using CDSA, in combination with commercial electromagnetic software (on this occasion two programs called OPERA and MagNet, respectively, were used), are presented with the aim of aiding the design optimisation of electromagnetic devices. The two problems can be categorised as Topology Optimisation (TO) and Shape Optimum Design (SOD) and generally they are considered to be different from each other. However, from the viewpoint of deriving and implementing a design sensitivity formula, it is noticed that they follow a very similar procedure [2]. Thus, after minor modifications to a part of the optimization module of $\mathrm{TO}$, the same program architecture can be applied to SOD problems.
Finally, in order to verify the advantages of CDSA, the problems are tested by CDSA in conjunction with different EM software. In addition, with the view of facilitating understanding of the method itself, a more general and very common program architecture consisting of MS Excel spreadsheets and the Visual Basic (VB) editor is attempted.

\section{RESULTS}

\subsection{Topology Optimisation of a MRI}

Fig. 1 shows a quarter of a model of a magnet assembly for an MRI device where the residual flux density of the magnet is $1.21 \mathrm{~T}$. Although the actual assembly is three-dimensional, here it has been simplified to an axi-symmetric problem. The design goal is to find an optimized distribution of shimming magnets over the pole piece surface to obtain a homogenous field distribution in a $30 \mathrm{~cm}$ diameter spherical volume (DSV). The shimming magnet has a residual flux density of $0.22 \mathrm{~T}$ and thickness of $3 \mathrm{~mm}$ and the domain is subdivided into 120 regions.

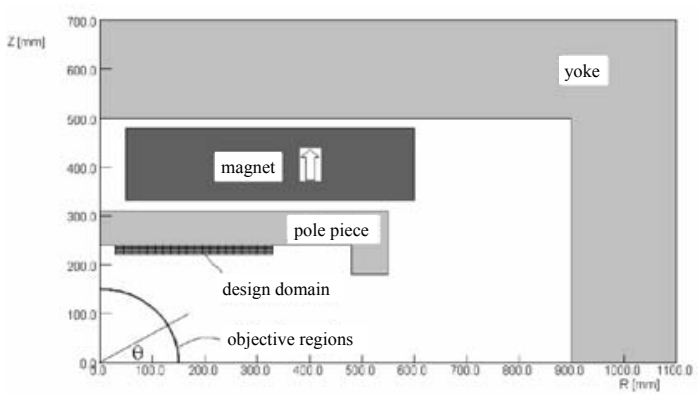

Fig. 1. A quarter model of an open permanent-magnet type MRI. 


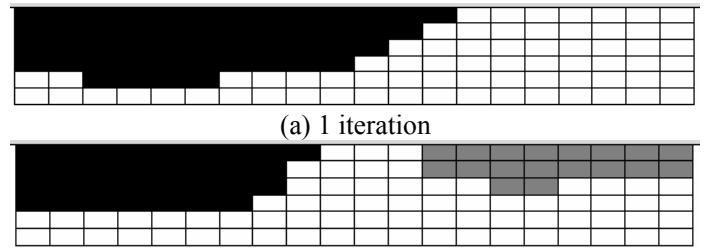

(b) 3 iterations

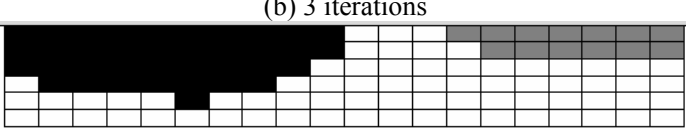

(c) 7 iterations

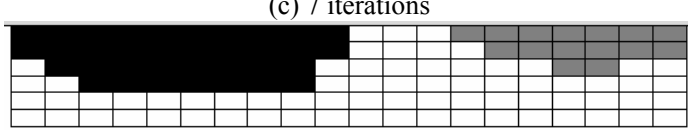

(d) 10 iterations

Fig. 2. Changes of shimming magnet distribution during optimization. (Black cell: $+\mathbf{M}_{\mathrm{s}}$, Gray cell: $-\mathbf{M}_{\mathrm{s}}$ )

The design domain to be occupied by materials is subdivided into multiple individual regions so that material properties can be imposed in each region prior to FE mesh generation. The individual regions correspond to design cells and a linear static OPERA$2 \mathrm{D}$ solver was used for analysis. The change of the shimming magnet distribution during the optimization is shown in Fig. 2 where the uniformity of the fields is improved 4 times compared with the initial design.

\subsection{Shape Optimisation of a Motor}

A BLDC motor with 8 permanent magnets and 12 salient stator poles is considered and optimization is carried out to minimize the cogging torque. The outer radii of the teeth, magnet and rotor yoke are $13.8 \mathrm{~mm}$, $15.3 \mathrm{~mm}$ and $16 \mathrm{~mm}$, respectively. The depth of the teeth is $2.5 \mathrm{~mm}$ whereas that of the magnet and yoke is $3.8 \mathrm{~mm}$. Only one-eighth of the problem needs to be modelled owing to symmetry (Fig. 3).

To investigate the fringing effect, an optimization of the pole face shape was performed using CDSA in conjunction with commercial software (MagNet 6). Design variables are defined at finite element vertices forming the outline of the stator pole, Fig. 4. To prevent a saw-toothed pole face shape, a cubic spline interpolation curve is introduced. Thus the movement of the 5 control points marked with dotted circles on the pole face in Fig. 4 constrains their corresponding 11 vertices to be positioned on a smooth curve. Due to the $15^{\circ}$ periodicity of the cogging torque, both $2 \mathrm{D}$ and $3 \mathrm{D}$ nonlinear FE analyses are carried out every $1.5^{\circ}$ from $0^{\circ}$ to $15^{\circ}$. The design details necessary for shape optimisation are contained in MS Excel spreadsheets and VB editor communicating with standard FE analysis software. After creating a 3D model according to the $2 \mathrm{D}$ optimized pole shape, a comparison of the cogging torque waveforms obtained from the 2D and 3D optimised pole shapes is shown in Fig. 5. It is revealed that the $3 \mathrm{D}$ optimised pole reduces the cogging torque by $25 \%$ of the initial value whereas the $2 \mathrm{D}$ analysis suggests only a $13 \%$ reduction due to the neglected fringing effect.

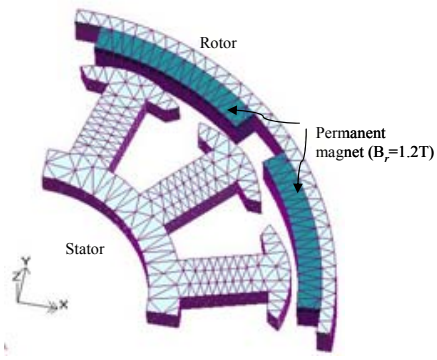

Fig. 3. 3D model used for optimisation.

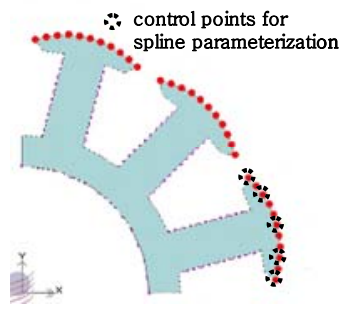

(a) 33 vertices in $2 \mathrm{D}$

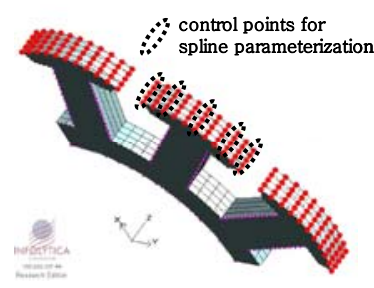

(b) 165 vertices in $3 \mathrm{D}$
Fig. 4. Design variables in 2D and 3D optimisation.

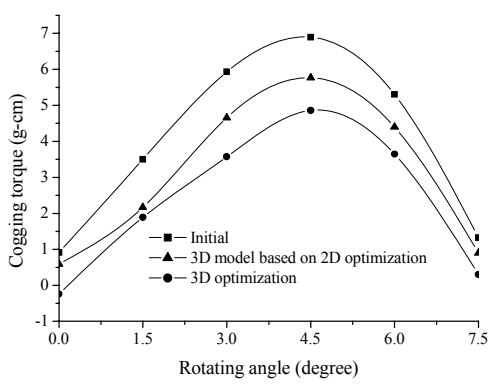

Fig. 5. Cogging torque waveforms for the initial, $2 \mathrm{D}$ and $3 \mathrm{D}$ optimal shapes.

\section{CONCLUSIONS}

The results show that CDSA is a very efficient optimisation technique offering big savings on computational effort due to the fact that computing times do not depend on the number of design variables. A flexible program architecture, combining MS Excel spreadsheets and Visual Basic script with a standard FE software package, proposed with the aim of aiding the efficient design optimisation, will be described in the extended version of the paper.

\section{REFERENCES}

[1] Kim D., Ship K., and Sykulski J.: Applying continuum design sensitivity analysis combined with standard EM software to shape optimisation in magnetostatic problems, IEEE Trans. Magn., 2004, 40, pp. 1156 - 1159

[2] Kim D., Sykulski J, and Lowther D.: A novel scheme for material updating in source distribution optimization of magnetic devices using sensitivity analysis, IEEE Trans. Magn., 2005, 41, pp. $1752-1755$ 